\title{
Editorial: intelligent GIServices
}

\author{
Peng Yue $^{1} \cdot$ Rahul Ramachandran ${ }^{2} \cdot$ Peter Baumann $^{3}$
}

Published online: 21 August 2015

(C) Springer-Verlag Berlin Heidelberg 2015

Web Service technologies are being actively used in Earth Science and consequently Geographical Information Systems (GIS) are moving from GISystems to GIServices. Web Services are the key technology component for establishing distributed geospatial data and information infrastructure such as Spatial Data and Information Infrastructure, e-Science, and Cyberinfrastructure. Traditional geosciences applications and systems are developed and used in siloed environments. However, in a distributed information infrastructure, data and high-level geospatial information products are served by distributed geospatial data services, transformed by dynamic processing services and workflows, and consumed by smart clients.

In addition to the shift to distributed architecture, geospatial domain is also experiencing an explosive growth in both volume and variety of data being gathered. Development of tools to fully explore and analyze these datasets has not kept pace with this growth. With the current focus on Big Earth Data, methods for utilizing processing services to analyze large volumes of distributed heterogeneous geospatial data are now being actively investigated. Intelligent GIServices will en-

Published in the Special Issue of Intelligent GIServices with Guest Editors Dr. Peng Yue, Dr. Rahul Ramachandran and Dr. Peter Baumann

Peng Yue

pyue@whu.edu.cn

1 State Key Laboratory of Information Engineering in Surveying, Mapping and Remote Sensing (LIESMARS), Wuhan University, 129 Luoyu Road, Wuhan, Hubei 430079, China

2 NASA/Marshall Space Flight Center, Huntsville, AL 35812, USA

3 Jacobs University Bremen, Bremen, Germany compass various aspects of GIService provisioning and use including discovery, selection, chaining, and invocation-all with the end goal of delivering geospatial information and knowledge to the end users.

This special issue on intelligent GIServices presents stateof-the-art technologies and approaches on GIServices. Five papers have been accepted for this publication.

The paper authored by Yue et al. (2015) provides an overview of intelligent GIServices. The concept of intelligent GIServices is presented, followed by a review of relevant technologies. A list of key research issues is highlighted, together with possible solutions. It is envisioned that integrating emerging AI technologies and GIServices will lead to better services to solve multitude of GIS problems. Infrastructure tools and APIs will lower the technical threshold in using distributed GIServices, and facilitate the easy integration of distributed geospatial resources. The paper authored by Liu et al. (2015) in this special issue adopts a light-weight mashup approach by designing Web APIs for sea ice data. The approach can provide a flexible and easy-to-use way for online sea ice data sharing, visualization and spatio-temporal analyses. Semantics is a fundamental issue in developing intelligent GIServices. The paper authored by Zhang et al. (2014) focuses on the performance of semantic processing, and presents a map-reduce based parallel approach for improving the query performance of GeoSPARQL. Intelligent GIServices can learn from geospatial problem solving, such as choosing the best services or workflows for a specific task. The paper authored by $\mathrm{Hu}$ et al. (2015) proposes a task-oriented approach for intelligent acting of GIServices using Sensor Web services and Quality of Services (QoS). The tasks facilitate the expression of users' requirements and capture problem-solving knowledge. The last paper, authored 
by Peng et al. (2015) is an application of GIServices in the agro-geoinformatic domain. It demonstrates how GIServices can be used to provide on-demand agricultural drought analysis, and facilitates the perception process in agricultural drought management. It also serves as an exemplar for other applications of GISservices.

As the guest editors of this special issue, we thank all the authors for contributing their papers and the reviewers for their valuable reviews and comments. We are also grateful to the Editor-in-Chief Dr. Hassan A. Babaie for the assistance on this issue. We hope that this special issue could help to promote the research on providing better GIServices and serve as a valuable resource to the ESI community interested in GIServices.

\section{References}

Hu L, Yue P, Zhang M, Gong J, Jiang L, Zhang X (2015) Task-oriented Sensor Web data processing for environmental monitoring. Earth Sci Inf. doi:10.1007/s12145-015-0235-1

Liu X, Li S, HuangW, Gong J (2015) Designing sea ice web APIs for ice information services. Earth Sci Inf. doi:10.1007/s12145-015-0207-5

Peng C, Deng M, Di L, Han W (2015) Delivery of agricultural drought information via web services. Earth Sci Inf. doi:10.1007/s12145014-0198-7

Yue P, Baumann P, Bugbee K, Jiang L (2015) Towards intelligent GIServices. Earth Sci Inf. doi:10.1007/s12145-015-0229-Z

Zhang C, Zhao T, Anselin L, Li W, Chen K (2014) A map-reduce based parallel approach for improving query performance in a geospatial semantic web for disaster response. Earth Sci Inf. doi:10.1007/ s12145-014-0179-x 